Lymphology 52 (2019) 61-70

\title{
EVALUATING THE RESPONSIVENESS OF THE FULLERTON ADVANCED BALANCE SCALE IN PATIENTS WITH LYMPHEDEMA SECONDARY TO BREAST CANCER SURGERY
}

\author{
A.K. Yoosefinejad, M. Hadadi, P. Eslamloo
}

Physical Therapy Department (AKY) and Orthotics and Prosthetics Department (MH), School of Rehabilitation Sciences; Rehabilitation Sciences Research Center (AKY,MH) and Student Research Committee (PE), Shiraz University of Medical Sciences, Shiraz, Iran

\section{ABSTRACT}

Lymphedema following surgical treatment for breast cancer can impair balance and predispose patients to falling. Fullerton Advanced Balance (FAB) Scale is a reliable and valid tool which can identify persons with different balance levels, but its responsiveness has not been investigated in patients with lymphedema secondary to breast surgery. Thirty women with stage 2 lymphedema secondary to breast cancer treatment received complex decongestive therapy (CDT) for 2 weeks as a routine treatment method. They were evaluated with FAB Scale and Timed Up and Go Test (TUGT), volumetric measurements and circumferential measurements of the upper limbs before and after CDT. A moderate change was found in FAB score after CDT (Cohen's effect size =0.65). For FAB, the computed standard error of the mean was 0.85 and minimal detectable change was 2.33. Significant improvement in FAB score and TUGT results, and significant reductions in circumferential and volumetric measurements were seen after 2 weeks of CDT. The FAB score change showed a moderate correlation with circumference change and volumetric change $(r=-0.41)$ but a very weak correlation with TUGT change $(r=-0.1)$. The FAB Scale showed acceptable responsiveness in detecting treatment effects in patients with unilateral secondary lymphedema after breast cancer treatment.

Keywords: breast cancer, lymphedema, Fullerton Advanced Balance Scale, Timed Up and Go Test, balance, responsiveness

Lymphedema is a chronic, progressive complication following surgical interventions used in the treatment of breast cancer (1). The most common timeframe for the appearance of lymphedema is within the first 12 months post-surgery (2); nevertheless, it can develop up to 20 years later (2). Lymphedema can cause both physical and psychological complications. Among the former are limitations in motion, decreased strength and performance, and balance impairment (3). Reduced directional control and increased postural sway due to displacement of the center of gravity toward the lymphedema side have also been reported in these patients. Altered weight distribution and limb asymmetry following lymphedema can impair balance and predispose the patients to falling (3). Basar et al showed that asymmetric fluid distribution in the upper extremities can increase postural sway and the risk of falls in a group of women with unilateral secondary lymphedema following breast cancer (4). 
The detection of balance impairments and information on the efficiency of a specific intervention in improving balance deficits can be helpful in planning the treatment protocol. Various scales are currently used to evaluate balance in different group of patients. The Fullerton Advanced Balance (FAB) Scale, developed by Rose et al, is a relatively new performance-based measure specifically designed to evaluate balance in independent functioning older adults $(5,6)$. It is a reliable and valid tool which can discriminate among participants with different balance levels (7). This scale was shown to be valid for predicting falls in community-dwelling adults (8). Moreover, it had excellent test-retest (interclass correlation coefficient $=0.98$ ) and inter-rater reliability (interclass correlation coefficient $=0.98$ ) in patients with breast cancer after chemotherapy (9).

Despite its acceptable psychometric properties, previous studies have yielded discrepant results regarding the use of the FAB Scale as a screening tool in different groups. Schlenstedt et al showed that the FAB Scale was a better detector of changes in the postural control system in patients with Parkinson disease than the Mini-Balance Evaluation Systems Test and Berg Balance Scale (10). In contrast, Kim showed that the FAB Scale was not a suitable instrument to predict the risk of falls in children with cerebral palsy (11).

The efficacy of an instrument to detect changes after treatment can be evaluated by an index known as responsiveness (12), which is an aspect of construct validity (13). It is defined as the ability of an instrument to detect changes over time in the construct to be measured (14). It can also be based on the application of a treatment method of known efficacy (15). The responsiveness of the FAB Scale has not been evaluated to date.

Several nonsurgical methods are used to manage lymphedema (16). Some of these methods include compression therapy, advanced pneumatic compression pumps, exercise, and complex (or complete) decongestive therapy (CDT) (16). This last option is cur- rently the standard treatment for lymphedema (17).

To the best of our knowledge, the responsiveness of the FAB Scale has not been investigated in patients with lymphedema secondary to breast cancer surgery. Moreover, the effects of CDT on improving balance in patients with lymphedema are unknown. The objective of this study was to evaluate the responsiveness of the FAB Scale in a group of women with unilateral secondary lymphedema after breast cancer treatment.

\section{MATERIALS AND METHODS}

This cross-sectional study was conducted between May 2017 and February 2018 at the lymphedema clinic of Motahari Hospital, Shiraz University of Medical Sciences, Shiraz, Iran. The study was approved by the ethics committee of Shiraz University of Medical Sciences in accordance with the standards of the Helsinki declaration.

A sample of 30 women with unilateral secondary lymphedema after breast cancer treatment, based on a pilot study, was recruited. All the participants provided their informed consent in writing before the beginning of the study. All the subjects underwent mastectomy and also all underwent radiation therapy. The axillary node sampling among the participants ranged from 3 to 39 . The inclusion criteria were age between 18 and 65 years, and stage 2 lymphedema. According to the International Society of Lymphology (ISL), stage 2 lymphedema is defined as pitting with a moderate degree of lymphedema which is not alleviated by elevating the limb (18). A moderate degree of lymphedema, consistent with stage 2 lymphedema according to the ISL, is defined as an absolute circumference difference of more than $2 \mathrm{~cm}$ in the upper extremities (19). The volume discrepancy between upper limbs is considered $200 \mathrm{~mL}$ for a moderate degree of lymphedema (19).

The exclusion criteria were orthopedic surgery in the lower limbs, inflammatory arthritis, uncorrected visual problems, throm- 
bophlebitis in the lower limbs, bilateral breast cancer treatment or bilateral mastectomy, coronary heart disease, central or peripheral nervous system disorders, spinal column surgery, consumption of alcohol or caffeine during the previous 24 hours, skin lesions with a risk of infection during volumetric measurements, and consumption of any medication that might have negative effects on balance.

Patients who met the inclusion criteria and had no exclusion criterion were evaluated with the FAB Scale and Timed Up and Go Test (TUGT). In addition, volumetric measurements of the upper limbs and circumferential measurements of different specified parts of the upper limbs were recorded.

The FAB Scale was first developed by Rose et al (6) to evaluate balance in higher functioning older adults. This scale comprises 10 items evaluating several aspects of balance. Five probable responses are considered for each item, and the items are scored between 0 (the worst possible response) and 4 (the best possible response). The maximum score on the scale is 40 , and the greater the score, the better the balance. Scores \pm 25 are interpreted as balance impairment and risk of falling.

The TUGT was developed by Podsialdo et al as a reliable test to evaluate mobility and dynamic balance, and also as an index to predict falling $(6,20)$. The patients sat on an adjustable chair without arm rests. Then they were asked to stand up and walk toward a cross located on the floor 3 meters ahead, turn around and return to the chair. Walking speed was self-paced. Each patient performed the TUGT three times and the average time was recorded for final analysis. A time longer than $\mathbf{1 3 . 5}$ seconds indicates a high risk of falling.

Circumferential measurements are used to estimate limb edema. The reliability of this method ranged between 0.96 and 0.99 in women with upper extremity lymphedema (21). Arm circumference measurements were made at different distances from the fingertips using a thin, flexible tape. The underlying landmarks selected for circumference measurements were the mid-ulnar styloid process, middle of the forearm (between the styloid process and elbow joint), elbow joint (olecranon), middle of the upper arm (between the elbow joint and acromion), and $65 \%$ of the distance between the olecranon and acromion (22). To obtain a better estimate of circumferential changes following CDT at distal potions of the upper extremity, two distal points were measured: one at the metacarpal-phalangeal (MCP) joint and another at the first web space (web).

Volumetric measurement is an accessible, inexpensive, hygienic, easy to use, and reliable method that provides valuable information on limb shape (23). Water displacement is considered a gold standard according to the principle of Archimedes $(21,24)$. The temperature of water in the container was kept constant at $20^{\circ} \mathrm{C}$. The upper limb was submerged in the container to a level $65 \%$ of the distance between the elbow (olecranon) and shoulder tip (acromion) (22). The same level was used for the final volumetric measurements. Limb volume change was defined as the difference in the affected upper limb between the baseline and post-treatment values.

In the next stage, each patient received CDT for 2 weeks, 5 days a week. This therapy is commonly used in the management of lymphedema, and consists of a combination of skin care, manual lymphatic drainage, exercise, and compression therapy with multilayer bandaging (25). In the present study, CDT consisted of four components:

1. Protecting the skin and nails. Patients with lymphedema are prone to infections such as cellulitis. In addition, wounds and abrasions may occur secondary to skin extensibility. These problems may lead to further lymphedema. Patients were asked to keep edematous areas clean, dry, and moisturized with a suitable emollient. They were also advised to observe the skin in specified intervals (26).

2. Manual lymphatic drainage (MLD) is a major component of CDT. The aim of MLD is to redirect lymph across the plexus of initial lymphatics toward healthy lymph 
nodes and allow the fluid to enter the system. It is applied to regional lymph nodes and lymph vessels proximal to trauma for $15-20$ minutes (27). This technique uses rhythmic, purposeful, mild massaging with the fingertips in stationary circular movements (27). First, cervical and supraclavicular lymph nodes were drained; then the lymph nodes of the axilla, abdominal and inguinal regions were drained. This was followed by drainage of the lymph vessels among the main nodes. After that, centrifugal massaging was done on the upper limb including the arm, elbow, forearm and hand. The accumulated lymph was thus centripetally directed to the sound side.

3. Compression bandaging is used to maintain the effects of MLD and reduce lymph accumulation. Patients were instructed to use bandaging for 23 hours a day (27), in order to compress the lymphatic vessels between the muscle and the compression bandage, causing them to be pumped manually. Tubular bandages were first placed directly on the skin. Then compression bandages were applied with an even $30 \%-40 \%$ pre-stretch and 50\%-70\% overlap while the patient was seated (27). The bandage consisted of different layers including soft foam rolls (Rosidal soft) to pad the hand and arm, synthetic cotton (Cellona) to pad the elbow, wrist and hand areas, and gauze bandages (Mollelast) to keep the padding in place.

4. Exercise, to facilitate venous and lymphatic flow (28). This part of CDT consisted of different exercises to improve lymph passage, reduce edema, and maintain joint range of motion and muscle strength. The exercise program included active, repetitive, and nonresistive movements. Patients were instructed to perform the exercises while wearing their compression bandages.

After the treatment period, all measurements were recorded again. In addition, the patients were asked to complete a Global Rating of Change (GRC) Scale, a Likert-type scale that can be scored by patients or physicians. The score ranges from -7 (large deterioration) to +7 (large improvement), and a score of 0 indicates no change. Commonly, partici- pants who report either getting "a little better" or "a little worse" constitute the minimal change subgroups (29).

All data were analyzed with SPSS v. 21 (IBM Statistics, New York, NY, USA). Descriptive analyses were used for demographic characteristics. Floor and ceiling effects of FAB scores were computed for baseline and post-treatment assessments as the percentage of participants who obtained the minimum and maximum scores.

Responsiveness of the FAB Scale was evaluated with both anchor-based and distribution-based methods, to analyze the changes in score between baseline and the post-treatment assessments. Distribution-based statistics included Cohen's effect size (ES), standardized response mean (SRM), standard error of the mean (SEM), minimal detectable change (MDC), and paired t-tests. The ES and SRM express score change based on standard deviation (SD) estimates; both are standardized indices of power of an instrument or scale to detect true change. Cohen's ES was calculated with the following formula:

$$
\begin{aligned}
& \text { Cohen's effect size }=\text { Mean }{ }_{\text {post-treatment }}{ }^{-} \\
& \text {Mean }{ }_{\text {pre-treatment }} / \mathrm{SD}_{\text {pre-treatment }} \\
& \text { The SRM was calculated as Mean }{ }_{\text {post- }} \\
& \text { treatment }- \text { Mean }{ }_{\text {pre-treatment }} / \mathrm{SD}_{\text {post-treatment }}-\mathrm{SD}^{-}
\end{aligned}
$$
pre- treatment ${ }^{\circ}$

An ES $\leq 0.2$ indicates a small change, an ES of 0.3-0.7 reflects moderate change, and an ES $\geq 0.8$ reflects a large change. To assess SRM with the same values for ES, we used the adjusted SRM according to Cohen's threshold, which was calculated with the formula: SRM adjusted $=\mathrm{SRM} \times \sqrt{ } \mathbf{2} \times \sqrt{ } \mathbf{1}-\mathrm{r}$, where $\mathrm{r}$ is Pearson's correlation coefficient between pre- and post-treatment data. The SEM was calculated as $\mathrm{SEM}=\mathrm{SD} \times \sqrt{ } \mathbf{1}-\mathbf{r}$.

The $\mathrm{SD}$ was calculated as $\mathrm{SD}=\sqrt{ }(\mathrm{SD}$ post-treatment
$\times \mathrm{SD}_{\text {pre-treatment }}$ $\mathrm{P}_{\text {interclass correlation coeffi- }}$ cient (ICC).

The MDC was calculated as SEM $\times 1.96$ $\times \sqrt{ } 2$. Because MDC is better represented by a range of values, this index was calculated with two commonly used ES estimates: 0.3 
$\times \mathrm{SD}$
$\mathrm{SD}$ pre-treatment
is the standard deviation of $\mathrm{FAB}$ score before treatment. Changes in scores from paired t-test analyses were compared with 0.3 $\times \mathrm{SD}_{\text {pre-treatment }}$ and $0.5 \times \mathrm{SD}_{\text {pre-treatment }} \mathrm{MDC}$ estimates to determine whether statistically significant differences exceeded the MDC estimates for each measure.

Paired t-tests were used to determine whether significant post-treatment changes occurred in any of the variables considered in this study.

To evaluate anchor-based methods, we used both patient-based methods including GRC scale and TUGT results, and clinical anchors including volumetric and circumferential measurements of predefined parts of the upper limbs. Pearson's correlation coefficient was calculated for score changes in all variables, and for FAB score changes. The $r$ values were categorized as follows: $\mathbf{0 - 0 . 1 9 =}$ very weak correlation, $0.2-0.39=$ weak correlation, 0.4-0.69 = moderate correlation, $0.7-0.89=$

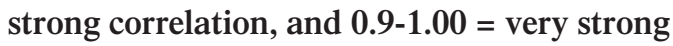
correlation (30).

\section{RESULTS}

Demographic data of the participants are summarized in Table 1. No floor effect was noted for baseline measures $(3.3 \%)$ or the secondary assessment $(6.7 \%)$ of FAB scores, but a ceiling effect was observed both at baseline $(20 \%)$ and in the secondary assessment (43\%). The computed Cohen's ES was 0.65 , reflecting a moderate change in $\mathrm{FAB}$ score after CDT. The adjusted SRM was computed as 0.59 , reflecting a moderate change in $F A B$ score after treatment. The computed SEM and MDC were 0.85 and 2.33, respectively. The estimated 0.3 and 0.5 SDs were 1.09 and 1.82 , respectively.

The results indicated that FAB score after 2 weeks of CDT improved significantly in comparison to baseline. The TUGT times also improved significantly. Moreover, significant reductions were seen in all circumferential measurements in the post treatment evalua-
TABLE 1

Demographic Data of the Participants

\begin{tabular}{|ll|}
\hline Characteristic & $\begin{array}{l}\text { Participants } \\
\text { (n=30) } \\
\text { mean } \pm \text { SD }\end{array}$ \\
\hline Age (years) & $50.53 \pm 8.95$ \\
Height (cm) & $156.06 \pm .18$ \\
Weight (kg) & $74 \pm 10.68$ \\
BMI (kg/m²) & $30.41 \pm 4.33$ \\
Hand dominance & Right: 27, \\
Time since surgery (years) & $4.80 \pm 4.77$ \\
Onset of lymphedema years) & $4.26 \pm 4.82$ \\
\hline
\end{tabular}

tion on the affected side, except for the second arm point $(\mathrm{p}=0.32)$. Measurements on the unaffected side showed no statistically significant differences in the post treatment assessment compared to pre-treatment values. Volumetric measurements and calculations showed that edema was reduced by $272 \mathrm{~mL}$ after 2 weeks of CDT. The comparisons of pre- and posttreatment values for all variables are summarized in Table 2.

The correlation between FAB score change and TUGT change was very weak $(\mathrm{r}=\mathbf{- 0 . 1}, \mathrm{p}=0.53)$. A moderate negative correlation was observed $(\mathrm{r}=\mathbf{- 0 . 4 1}, \mathrm{p}=\mathbf{0 . 0 2})$ between volumetric measurement change and FAB score change. The correlation between FAB score change and circumference change in the mid arm was moderate $(r=$ $-0.58, p=0.001)$. A weak correlation $(r=-0.33$, $\mathrm{p}=0.07$ ) was seen between FAB score change and circumference change in the elbow. A moderate negative correlation was observed between FAB score change and circumference change in the mid elbow $(r=-0.51, p=0.004)$. The correlation between FAB score change and web circumference change $(r=-0.18$, $\mathrm{p}=0.32)$ and between FAB score change and MCP circumference change $(\mathrm{r}-0.25, \mathrm{p}=0.18)$ was weak. A weak negative correlation was found between FAB score change and ulna 


\begin{tabular}{|c|c|c|c|c|}
\hline Variable & Side & $\begin{array}{l}\text { pre-treatment } \\
(\text { mean } \pm \text { SD })\end{array}$ & $\begin{array}{l}\text { post-treatment } \\
(\text { mean } \pm \text { SD) }\end{array}$ & p-value \\
\hline Metacarpal-phalangeal joint $(\mathrm{cm})$ & $\begin{array}{l}\text { affected } \\
\text { unaffected }\end{array}$ & $\begin{array}{l}17.65 \pm 1.74 \\
16.67 \pm 1.04\end{array}$ & $\begin{array}{l}16.72 \pm 1.08 \\
16.64 \pm .89\end{array}$ & $\begin{array}{l}<0.001 \\
0.75\end{array}$ \\
\hline Web (cm) & $\begin{array}{l}\text { affected } \\
\text { unaffected }\end{array}$ & $\begin{array}{l}20.18 \pm 2.67 \\
18.61 \pm 1.25\end{array}$ & $\begin{array}{l}19.23 \pm 1.28 \\
18.71 \pm 1.12\end{array}$ & $\begin{array}{l}\mathbf{0 . 0 1} \\
0.37\end{array}$ \\
\hline Styloid of ulna $(\mathrm{cm})$ & $\begin{array}{l}\text { affected } \\
\text { unaffected }\end{array}$ & $\begin{array}{l}17.63 \pm 2.86 \\
15.95 \pm 1.33\end{array}$ & $\begin{array}{l}16.92 \pm 1.56 \\
15.85 \pm 1.02\end{array}$ & $\begin{array}{l}\text { 0.04 } \\
0.48\end{array}$ \\
\hline Mid forearm $(\mathrm{cm})$ & $\begin{array}{l}\text { affected } \\
\text { unaffected }\end{array}$ & $\begin{array}{l}26.70 \pm 3.74 \\
22.47 \pm 2.54\end{array}$ & $\begin{array}{l}25.26 \pm 2.65 \\
22.29 \pm 2.25\end{array}$ & $\begin{array}{l}<0.001 \\
0.24\end{array}$ \\
\hline Elbow (cm) & $\begin{array}{l}\text { affected } \\
\text { unaffected }\end{array}$ & $\begin{array}{l}28.75 \pm 3.61 \\
25.18 \pm 2.33\end{array}$ & $\begin{array}{l}27.77 \pm 2.92 \\
25.35 \pm 2.36\end{array}$ & $\begin{array}{l}\text { 0.004 } \\
0.26\end{array}$ \\
\hline Mid $\operatorname{arm}(\mathrm{cm})$ & $\begin{array}{l}\text { affected } \\
\text { unaffected }\end{array}$ & $\begin{array}{l}32.27 \pm 4.48 \\
29.08 \pm 3.24\end{array}$ & $\begin{array}{l}31.48 \pm 3.40 \\
29.32 \pm 3.34\end{array}$ & $\begin{array}{l}\mathbf{0 . 0 2} \\
0.36\end{array}$ \\
\hline Second arm point $(\mathrm{cm})^{*}$ & $\begin{array}{l}\text { affected } \\
\text { unaffected }\end{array}$ & $\begin{array}{l}32.50 \pm 3.78 \\
31.09 \pm 3.18\end{array}$ & $\begin{array}{l}32.73 \pm 3.57 \\
31.23 \pm 3.22\end{array}$ & $\begin{array}{l}0.32 \\
0.47\end{array}$ \\
\hline FAB Scale & & $35.76 \pm 3.64$ & $38.14 \pm 2.25$ & $<0.001$ \\
\hline TUGT (seconds) & & $11.70 \pm 2.42$ & $10.94 \pm 2.06$ & $<0.001$ \\
\hline Volumetric measurement $(\mathrm{mL})$ & & $673 \pm 554.49$ & $401 \pm 351.84$ & $<0.001$ \\
\hline
\end{tabular}

* $65 \%$ of the distance between the olecranon and acromion

circumference change $(r=-0.36, p=0.05)$.

Analysis of the patients' responses to the GRC Scale showed that $13(43.3 \%)$ of the participants became "better" and $16(53.3 \%)$ of them became "much better". Among all participants, only one $(3.3 \%)$ reported "no change" after 2 weeks of CDT. Comparisons of the mean changes in all variables between patients who reported feeling "better" versus "much better" in the GRC Scale are summarized in Table 3.

\section{DISCUSSION}

The FAB Scale is valid and reliable instrument basically designed to evaluate balance; it is currently used not only for older adults, but also in different groups such as children with cerebral palsy, patients with Parkinson's disease, and patients with breast cancer. Because this scale is expected to identify balance improvements after a given treatment, it is important to determine its responsiveness in different target populations. To our knowledge, the present study is the first to examine the responsiveness of the FAB Scale in patients with breast cancer treatment-induced lymphedema after CDT. For this purpose both distribution-based and anchor-based methods were used to estimate the changes in threshold values.

The findings showed a ceiling effect for the FAB Scale in patients with lymphedema. This may indicate a limited capability of the FAB Scale to identify patients with acceptable balance function.

Our analysis with distribution-based methods showed that the FAB Scale had a 


\begin{tabular}{|c|c|c|c|}
\hline \multicolumn{4}{|c|}{$\begin{array}{l}\text { TABLE } 3 \\
\text { Comparison of Mean Changes After } 2 \text { Weeks of CDT in Each Variable } \\
\text { Between Participants Who Became "Better" and Those Who Became } \\
\text { "Much Better" According to the Global Rating of Change Scale }\end{array}$} \\
\hline Variable & $\begin{array}{c}\text { "Better" }(n=13) \\
\text { Mean } \pm \text { SD }\end{array}$ & $\begin{array}{c}\text { "Much better" }(n=16) \\
\text { mean } \pm \text { SD }\end{array}$ & p-value \\
\hline FAB Scale & $1.95 \pm 2.05$ & $2.75 \pm 2.46$ & 0.36 \\
\hline TUGT (seconds) & $0.99 \pm 1.06$ & $0.56 \pm 0.85$ & 0.24 \\
\hline volume $(\mathrm{mL})$ & $189.23 \pm 314.97$ & $352.5 \pm 248.71$ & 0.13 \\
\hline Metacarpal-phalangeal joint $(\mathrm{cm})$ & $0.67 \pm 0.87$ & $1.12 \pm 1.06$ & 0.24 \\
\hline Web (cm) & $0.35 \pm 1.77$ & $1.47(2.09$ & 0.14 \\
\hline Styloid process of ulna $(\mathrm{cm})$ & $0.28 \pm 2.00$ & $1.10 \pm 1.93$ & 0.27 \\
\hline Mid forearm $(\mathrm{cm})$ & $0.62 \pm 1.36$ & $2.13 \pm 2.05$ & 0.03 \\
\hline Elbow (cm) & $0.77 \pm 1.65$ & $1.18 \pm 1.88$ & 0.53 \\
\hline Mid arm (cm) & $0.01 \pm 1.81$ & $1.45 \pm 1.81$ & 0.04 \\
\hline Second arm point $(\mathrm{cm})^{*}$ & $0.42 \pm 1.13$ & $0.21 \pm 1.16$ & 0.63 \\
\hline
\end{tabular}

* $65 \%$ of the distance between the olecranon and acromion

moderate ES and SRM: both values were in the medium range for score changes. The SRM reflects variability in the change of interest, and is an appropriate index to assess responsiveness because it is independent of sample size (31). This statistic indicated that FAB had moderate power to detect the changes that occurred after treatment in the group of patients studied here. The results for ES and SRM thus show a moderate degree of responsiveness for the FAB Scale.

Considering SEM value, the smallest change in the FAB Scale score that could indicate clinical differences between pre- and post-treatment measurements was calculated as $\mathbf{0 . 8 5}$. In addition MDC, an estimate of minimum acceptable change (without interference from measurement error) and thus an index of real change, was calculated as $\mathbf{2 . 3 3}$ for the FAB Scale. The range of possible FAB scores from 0 to 40 reflects the potentially high sensitivity of scale. The MDC was less than $6 \%$ of the total score. The calculated values SEM and MDC were lower than the changes in FAB score after treatment. The threshold for indicating clinically important differences in FAB scores for patients with lymphedema was approximately 2.33 points. The difference between pre- and post-treatment values for the same sample should be higher than the minimal important difference (MID) values to show true changes in the patients' status. However, our analysis showed that the FAB score change exceeded both estimated MID values. Because we are unaware of other studies designed to evaluate FAB Scale responsiveness, direct comparisons with earlier studies were not possible. 
The FAB score, like other measures analyzed in the present study, showed a significant change in post-intervention values. The post-treatment scores indicated improvement in the participants' health status after CDT. This may reflect the ability of the FAB Scale to detect balance changes after CDT in patients with lymphedema.

Regarding anchor-based methods, we analyzed the correlation of FAB scores with the clinical measures TUGT results, volumetric, and circumferential values. The results revealed a weak correlation with TUGT and circumferential measures in four areas, and a moderate correlation with volumetric value and circumferential measures in two areas. Because of the lack of specific measures to assess balance in patients with lymphedema, these correlations might be expected. The TUGT was originally developed for the older population, but our sample consisted of young women; therefore, TUGT might not be sensitive enough to detect changes in this latter population. Although all measures indicated changes in our group of patients, they had a weak to moderate correlation with FAB Scale scores. The highest correlation was found between FAB score change and circumference change in the mid arm and mid elbow; the lowest correlation was observed between FAB score change and TUGT change.

All participants, except one, reported feeling better or much better after CDT according to the GRC Scale results. Because none of the participants reported feeling "a little better" or a "little worse," it was not possible to use the anchor-based method with the GRC Scale data. Except for TUGT, all other variables improved more in the group who reported feeling "much better" after CDT than in the group who reported feeling "better." However, circumferential measurements in the mid elbow and mid arm differed significantly after treatment compared to baseline data.

This study had some limitations. First, TUGT was initially designed for older adults. Because of the lack of a gold standard for evaluating balance in patients with lymphede- ma, we used the TUGT as an index to investigate dynamic balance. Second, the results we obtained can only be generalized to patients with unilateral secondary lymphedema after breast cancer treatment, not to other groups with lymphedema or other types of cancer. We did not evaluate the presence and the extent of fibrosis due to secondary lymphedema in our study. Fibrosis might have influenced the treatment results.

\section{CONCLUSION}

In conclusion, the FAB Scale demonstrated acceptable responsiveness in detecting treatment effects in women with unilateral secondary lymphedema after breast cancer treatment. It showed a moderate correlation with changes in circumferential measurements in the mid elbow and mid arm. This scale can be used to evaluate balance in patients with lymphedema secondary to breast cancer treatment before and after therapy.

\section{ACKNOWLEDGMENTS}

This work was supported by a grant from the Vice Chancellory of Shiraz University of Medical Sciences (Grant Number: 95-01-0613100). We thank the patients for their kind participation in the study, and K. Shashok (AuthorAID in the Eastern Mediterranean) for improving the use of English in the manuscript.

\section{CONFLICT OF INTEREST AND DISCLOSURE}

The author declares no competing financial interests exist.

\section{REFERENCES}

1. Chiu, T: Management of secondary lymphoedema. Hong Kong Med. J. 20 (2014), 519-528.

2. Armer, J, B Stewart: Post breast cancer lymphedema: Incidence increases from 12 to 30 to 60 months. Lymphology 43 (2010), 118-127. 
3. Angin, S, D Karadibak, T Yavuzşen, et al: Unilateral upper extremity lymphedema deteriorates the postural stability in breast cancer survivors. Contemp. Oncol. 18 (2014), 279-284.

4. Basar, S, Y Bakar, I Keser, et al: Does lymphedema affect the postural stability in women after breast cancer? Topics in Geriatric Rehabilitation. 28 (2012), 287-294.

5. Hernandez, D, DJ Rose: Predicting which older adults will or will not fall using the Fullerton Advanced Balance scale. Arch. Phys. Med. Rehabil. 89 (2008), 2309-2315.

6. Rose, DJ, N Lucchese, LD Wiersma: Development of a multidimensional balance scale for use with functionally independent older adults. Arch. Phys. Med. Rehabil. 87 (2006), 14781485.

7. Klein, PJ, RC Fiedler, DJ Rose: Rasch analysis of the Fullerton Advanced Balance (FAB) scale. Physiother. Canada. 63 (2011), 115-125.

8. Jeon, Y-J, G-M Kim: Comparison of the Berg Balance Scale and Fullerton Advanced Balance Scale to predict falls in communitydwelling adults. J. Phy. Ther. Sci. 29 (2017), 232-234.

9. Wampler, MA, KS Topp, C Miaskowski, et al: Quantitative and clinical description of postural instability in women with breast cancer treated with taxane chemotherapy. Arch. Phys. Med. Rehabil. 88 (2007), 1002-1008.

10. Schlenstedt, C, S Brombacher, G Hartwigsen, et al: Comparing the Fullerton Advanced Balance Scale with the Mini-BESTest and Berg Balance Scale to assess postural control in patients with Parkinson disease. Arch. Phys. Med. Rehabil. 96 (2015), 218-225.

11. Kim, G-M: Comparison of the Pediatric Balance Scale and Fullerton Advanced Balance Scale for Predicting Falls in Children With Cerebral Palsy. Phys. Ther. Korea 23 (2016), 63-70.

12. Stratford, PW, JM Binkley, DL Riddle: Health status measures: Strategies and analytic methods for assessing change scores. Phys. Ther. 76 (1996), 1109-1123.

13. Revicki, D, RD Hays, D Cella, et al: Recommended methods for determining responsiveness and minimally important differences for patient-reported outcomes. J. Clin. Epidemiol. 61 (2008), 102-109.

14. Mokkink, LB, CB Terwee, DL Patrick, et al: The COSMIN study reached international consensus on taxonomy, terminology, and definitions of measurement properties for health-related patient reported outcomes. J. Clin. Epidemiol. 63 (2010), 737-745.
15. Revicki, DA, D Cella, RD Hays, et al: Responsiveness and minimal important differences for patient reported outcomes. Health Qual. Life Outcomes 4 (2006), 70.

16. Kayiran, O, C De La Cruz, $\mathrm{K}$ Tane, et al: Lymphedema: From diagnosis to treatment. Turkish J. Surg. 33 (2017), 51.

17. Zeltzer, AA, A Anzarut, M Hamdi: A review of lymphedema for the hand and upper-extremity surgeon. J. Hand Surg. Am. 43 (2018), 10161025.

18. The diagnosis and treatment of peripheral lymphedema: 2016 consensus document of the International Society of Lymphology. Lymphology 49 (2016) 170-184.

19. Cheville, AL, CL McGarvey, JA Petrek, et al: The grading of lymphedema in oncology clinical trials. Semin. Radiat. Oncol. 13 (2003), 214- 225.

20. Shumway Cook, A, S Brauer, M Woollacott: Predicting the probability for falls in community-dwelling older adults using the Timed Up \& Go Test. Phys. Ther. 80 (2000), 896-903.

21. Karges, JR, BE Mark, SJ Stikeleather, et al: Concurrent validity of upper-extremity volume estimates: Comparison of calculated volume derived from girth measurements and water displacement volume. Phy. Ther. 83 (2003), 134-145.

22. Taylor, R, UW Jayasinghe, L Koelmeyer, et al: Reliability and validity of arm volume measurements for assessment of lymphedema. Phy. Ther. 86 (2006), 205-214.

23. Armer, JM, BR Stewart: A comparison of four diagnostic criteria for lymphedema in a postbreast cancer population. Lymphat. Res. Biol. 3 (2005), 208-217.

24. Sagen A, R Kåresen, MA Risberg: The reliability of a simplified water displacement instrument: A method for measuring arm volume. Arch. Phys. Med. Rehabil. 86 (2005), 86-89.

25. Lasinski, BB (Ed.): Complete decongestive therapy for treatment of lymphedema. Semin. Oncol. Nurs. 29 (2013), 20-27.

26. Williams, A: Breast and trunk oedema after treatment for breast cancer. J. Lymphoedema 1 (2006), 3-39.

27. Zuther, J (Ed.): Lymphedema Management. Cancer Forum; 2005.

28. Hayes, S, H Reul-Hirche, J Turner: Exercise and secondary lymphedema: Safety, potential benefits, and research issues. Med. Sci. Sports Exerc. 41 (2009), 483-489.

29. Turner, D, HJ Schünemann, LE Griffith, et al: Using the entire cohort in the receiver operating characteristic analysis maximizes precision of the minimal important difference. J. Clin. Epidemiol. 62 (2009), 374-379. 
30. Farazdaghi, MR, A Mansoori, O Vosoughi, et al: Evaluation of the reliability and validity of the Persian version of Patient-Rated Elbow Evaluation questionnaire. Rheumatol. Int. 37 (20170, 743-750.

31. de Yebenes Prous, MJ, F Rodriguez Salvanes, L Carmona Ortells: [Responsiveness of outcome measures]. Rheumatol Clin. 4 (2008), 240-247.
Mohammad Hadadi, PhD

Chamran Blvd, Sheikh Abibvardi 1

School of Rehabilitation Sciences

P.O. Box 713451733

Shiraz University of Medical Sciences

Shiraz, Iran

Tel: +98-71-36271551

Fax: +98-71-36272495

E-mail: mhadadito@yahoo.com 